\title{
L'inondation du 30 novembre 1955 à Montpellier
}

\section{The floods at Montpellier on November 30th, 1955}

\author{
PAR PH. DEYMTÉ \\ INGÉNIEUR EN CHEF DES PONTS ET CHAUSSÉES
}

\begin{abstract}
Le 30 novembre 1955 , Montpellier a subi une inondation qui a fait trois victimes. Cette inondation a été causée, comme les inondations semblables antérieures, par une longue averse durant plusieurs heures, saturant le sol et présentant, dans sa phase la plus violente, une intensité de $0,73 \mathrm{~mm}$ par minute pendant 105 minutes.

La Tongueur de l'averse tombant sur un bassin court comme celui du Verdanson finit par étublir un régime permanent entre le débit tombé du ciel et le débit évacué par la rivière.
\end{abstract}

Le tableau ci-dessous indique la pluviosité d'un certain nombre de villes du Midi de la France et de Paris.

\begin{tabular}{|c|c|c|c|}
\hline $\begin{array}{c}\text { Lieu d'observation } \\
1\end{array}$ & $\begin{array}{l}\text { Moyenne } \\
\text { annuelle } \\
\text { en mm } \\
\quad 2\end{array}$ & $\begin{array}{c}\text { Nombre } \\
\text { moyen } \\
\text { de jours } \\
3\end{array}$ & $\mid \begin{array}{c}\text { Quotient } \\
\text { Col. 2 } \\
\text { Col. 3 } \\
4\end{array}$ \\
\hline 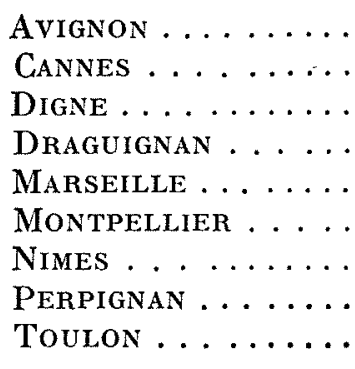 & $\begin{array}{l}616 \\
796 \\
775 \\
931 \\
572 \\
732 \\
691 \\
598 \\
733\end{array}$ & $\begin{array}{r}72 \\
82 \\
103 \\
92 \\
82 \\
94 \\
86 \\
80 \\
70\end{array}$ & $\begin{array}{r}8,55 \\
9,70 \\
7,40 \\
10,12 \\
6,98 \\
7,80 \\
8,00 \\
7,50 \\
10,50\end{array}$ \\
\hline $\begin{array}{l}\text { Paris } \ldots \ldots \ldots \ldots \\
\text { Carcassonne } \ldots \ldots \\
\text { Toulouse } \ldots \ldots \ldots\end{array}$ & $\begin{array}{l}607 \\
643 \\
684\end{array}$ & $\begin{array}{l}166 \\
145 \\
144\end{array}$ & $\begin{array}{l}3,66 \\
4,43 \\
4,75\end{array}$ \\
\hline
\end{tabular}

La moyenne annuelle de pluie est plus forte à Montpellier qu'à Paris; en outre cette moyenne

\begin{abstract}
On November 30th 1955 floods occured at Montpellier causing the loss of three lives. These floods and previons similar ones were caused by rain that fell for several hours saturating the ground and which at its most violent stage fell at a rate of $0.73 \mathrm{~mm}$ per minute for 105 minutes.

Such a long downpour falling on a short river basin like the Verdanson eventually sets up a steady regime between the falling rain and the flow of the river.
\end{abstract}

se répartit sur 94 jours seulement à Montpellier au lieu de 166 jours à Paris, de sorte que le quotient de la moyenne annuelle par le nombre de jours de pluie est de 7,8 à Montpellier et de 3,66 à Paris.

Le quotient correspondant de Toulouse et Carcassonne est plus voisin de celui de Paris que de celui de Montpellier ou des villes du littoral méditerranéen. Toulouse et Carcassonne sont en effet soumises comme Paris à l'influence de l'Océan Atlantique.

\section{***}

On sait que dans la région parisienne, pour la fréquence décennale, l'intensité moyenne $i=\mathrm{H} / \mathrm{T}$, au cours de la phase la plus violente d'une averse, est reliée à la durée $T$ de cette phase par la formule suivante :

$$
\frac{\mathrm{H}}{\mathrm{T}}=\frac{43}{\mathrm{~T}+10}(\text { GRISOLLET }),
$$

ou par l'expression monôme :

$$
\frac{\mathrm{H}}{\mathrm{T}}=11 \mathrm{~T}^{-0 . \tau} \text { (CAQUOT) }
$$

En utilisant cette relation et l'ensemble des données qui interviennent dans la formation des 
pointes de ruissellement, M. CAQuot a dégagé la formule suivante donnant le débit décennal en provenance d'une zone de construction, compte tenu de l'effet de capacité :

$$
\mathrm{Q}_{0}=1.340 \quad \mathrm{I}^{0,30} \quad \mathrm{C}^{1,17} \quad \mathrm{~A}^{0,75}
$$

$Q_{0}$ représentant le débit en litres par seconde;

$A$, la superficie du bassin versant en ha;

$C$, le coefficient de ruissellement moyen;

I, la pente moyenne sur le développement total du parcours de l'eau.

Pour Montpellier, les formules correspondantes établies par M. Grisollet et par M. Godard, directeur de la Station Météorologique de Montpellier, sont les suivantes :

$$
\begin{aligned}
& \frac{H}{T}=5,3 T^{-0,4} \\
& Q_{0}=580 \quad I^{0,16} C^{1,09} \quad A^{0,82} .
\end{aligned}
$$

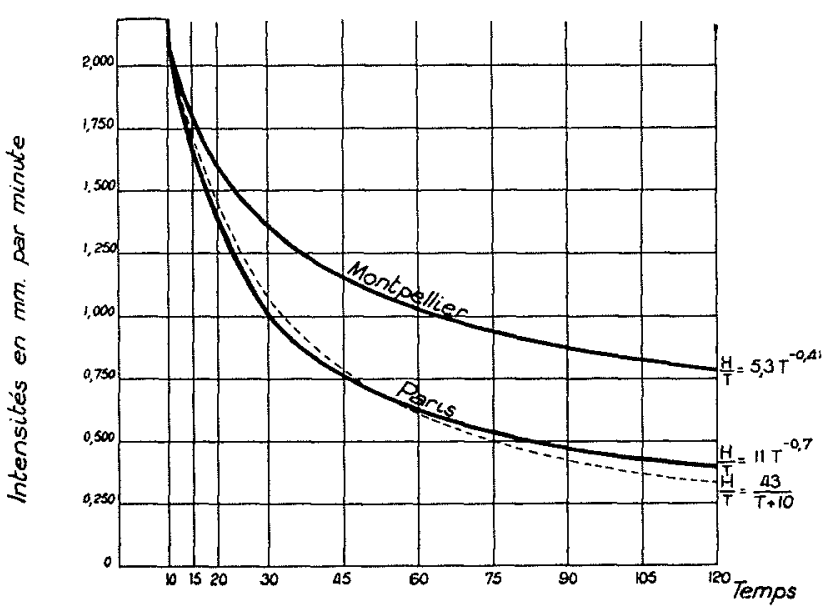

FIG. 1

La courbe $\mathrm{H} / \mathrm{T}$ de Montpellier est nettement au-dessus de la courbe correspondante de Paris, c'est-à-dire que, pour une même durée $T$, l'intensité est plus forte et l'averse plus soutenue (fig. 1).

L'inondation du 30 novembre 1955 à Montpellier, qui a fait trois victimes, est due à une averse exceptionnellement longue (15 heures) dont l'intensité a atteint $0,73 \mathrm{~mm}$ par minute pendant 105 minutes, de 17 h. 52 à 19 h. 37 , et succédant elle-même à une période pluvieuse, de sorte que le sol était déjà saturé. Même si cette saturation préalable n'avait pas été complète, la pluie tombée immédiatement avant la phase violente de l'averse, soit $52 \mathrm{~mm}$ pendant plus' de 8 heures, aurait suffi à achever cette saturation.

Cette longue averse a provoqué des débordements du Verdanson et l'engorgement de certains égouts, notamment de l'égout dit du ruisseau des Vaches.

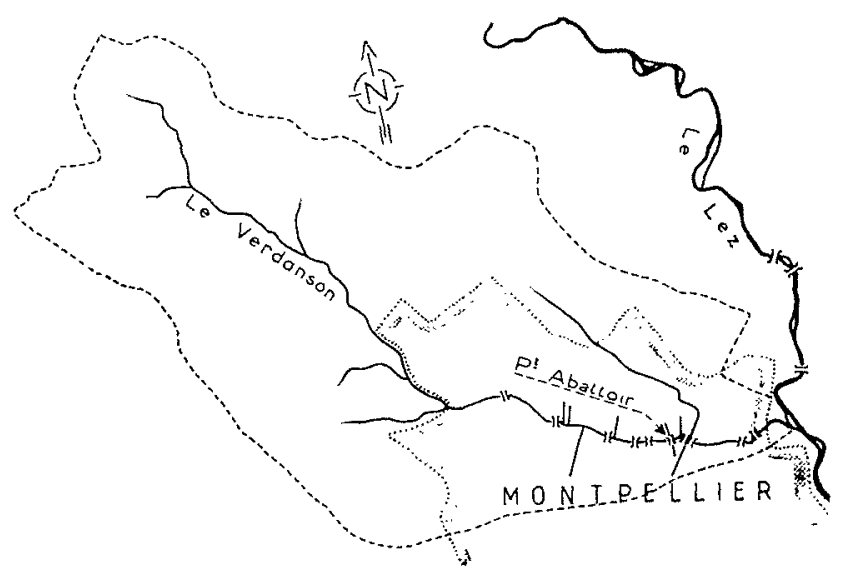

Fig. 2

Le Verdanson est un affluent du Lez, long de $20 \mathrm{~km}$ environ, qui traverse Montpellier et sert de collecteur général pour la partie nord-est de la ville. Son bassin versant total est de 1.600 ha environ, et son bassin versant à la sortie de la partie dense de l'agglomération (pont de la R.N. 113) de 1.350 ha environ (fig. 2).

Dans sa traversée de l'agglomération, le Verdanson est limité par des murs de quai en maconnerie de moellons et le fond du lit est bétonné. La plupart des nombreux ponts qui franchissent le Verdanson ont des débouchés très insuffisants. Le pont de l'Abattoir est le plus insuffisant (fig. 3).

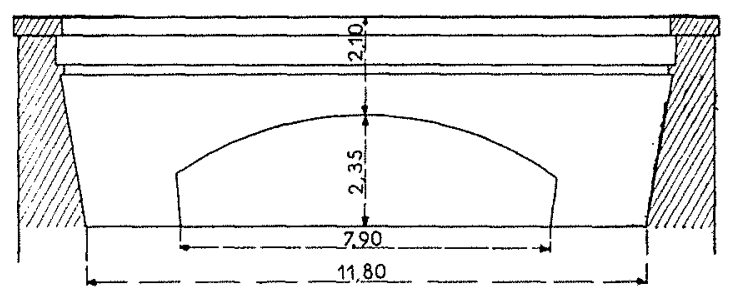

Fig. 3. - Pont de l'Abattoir

Si l'on se reporte en arrière, des débordements du Verdanson, causés également par des précipitations exceptionnellement longues, se sont produits à intervalles plus ou moins longs. Depuis un siècle, on a noté les inondations de $1862,1893,1907,1920$ et 1933. 
Le tableau ci-dessous résume les caractéristiques de ces longues averses :

\begin{tabular}{|c|c|c|c|c|}
\hline Averses & $\begin{array}{c}\begin{array}{c}\text { Hauteur } \\
\text { totale } \\
\text { de pluie }\end{array} \\
\\
\mathrm{mm}\end{array}$ & $\begin{array}{c}\text { Durée } \mathrm{T} \\
\text { de } \\
\text { l'averse } \\
\text { pendant } \\
\text { sft phase } \\
\text { la plus } \\
\text { violente } \\
\text { mn }\end{array}$ & $\begin{array}{c}\text { Hauteur } \\
\text { H } \\
\text { de pluie } \\
\text { tombée } \\
\text { pendant } \\
\text { la phase } \\
\text { la plus } \\
\text { violente T } \\
\text { mm }\end{array}$ & $\begin{array}{c}\begin{array}{c}\text { Intensité } \\
\text { de } \\
\text { la pluie } \\
\text { pendant } \\
\text { la durée T }\end{array} \\
\mathrm{mm} / \mathrm{mn}\end{array}$ \\
\hline 11 octobre 1862 : & 285 & 240 & 230 & 9,95 \\
\hline 29 août 1893 : & 186 & - & - & - \\
\hline 16 octobre $1907:$ & 111 & 60 & 41,8 & 0,70 \\
\hline 9 nov. 1907: & 77,6 & 60 & 45,9 & 0,76 \\
\hline 16 octobre 1920 : & 144 & 60 & 61,2 & 1,02 \\
\hline $26-27$ sept. 1933 : & 246,8 & 60 & 62,6 & 1,04 \\
\hline 30 nov. 1955 : & 148,4 & 105 & 76,6 & 0,73 \\
\hline
\end{tabular}

Les renseignements relatifs aux averses de 1862 et 1893 résultent de rapports plus ou moins précis et sont donnés sous toutes réserves. Ceux relatifs aux averses de 1907 résultent du relevé du pluviomètre installé à l'Ecole d'Agriculture de Montpellier, d'heure en heure. Ceux relatifs aux averses postérieures résultent du relevé du pluviomètre installé à la Station météorologique de Montpellier, de quart d'heure en quart d'heure.

Nous avons pu ainsi établir pour les averses de 1920,1933 et 1955 , la courbe donnant la hauteur de pluie en fonction du temps (fig. 4).

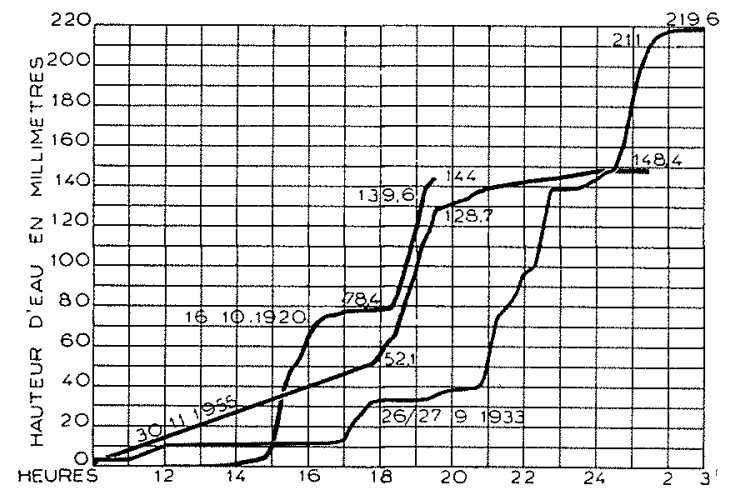

FIG. 4

Le débit d'une rivière qui a débordé est difficile à préciser; cependant, étant donné, d'une part, le peu d'étendue du bassin versant, d'autre part, la longueur des averses, on peut admettre que les débits maxima du Verdanson sont donnés par la formule :

$$
\mathrm{Q}=\mathrm{C} i \mathrm{~A}
$$

$C$ étant le coefficient de ruissellement;

$i$ l'intensité de l'averse pendant sa phase la plus violente;

A la surface du bassin versant.

En prenant pour le coefficient de ruissellement la valeur 0,9 justifiée par l'extrême saturation du sol au cours de ces longues averses, on trouve que le débit maximum du Verdanson à la sortie de la partie dense de l'agglomération varie de 140 à $200 \mathrm{~m}^{3}$ quand l'intensité de l'averse pendant sa phase violente varie de $0,77 \mathrm{~mm} / \mathrm{mn}$ à $1 \mathrm{~mm} / \mathrm{mn}$.

Le 30 novembre 1955 , le débit aurait ainsi atteint $146 \mathrm{~m}^{3}$ environ.

$$
Q_{0}=5801^{0,16} C^{1,09} A^{0,82}
$$
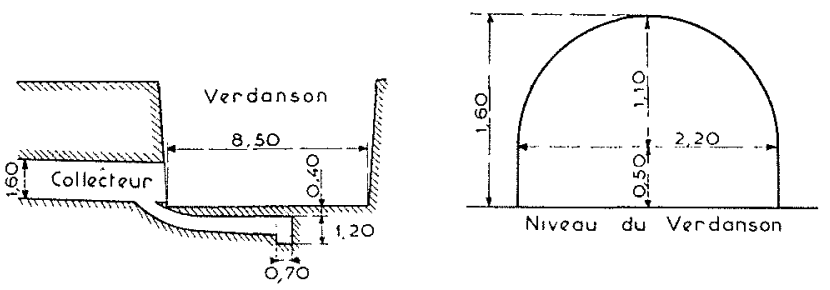

Fig. 5. - Egout du ruisseau des Vaches

Ce jour-là, le niveau de l'eau dans le Lez n'était heureusement pas élevé (cote : 0,40 à l'échelle des Quatre-Canaux), sinon les débordements du Verdanson auraient été encore plus importants.

Le nombre réduit des précipitations observées ne permet pas d'établir la fréquence de ces précipitations avec une certaine exactitude. Il semble cependant que cette fréquence soit voisine de 15 années.

En période sèche, les égouts déversent leurs eaux usées dans un collecteur situé sous le lit du Verdanson, de sorte que ce ruisseau est normalement à sec.

Un égout important est celui du ruisseau des Vaches; il aboutit au Verdanson par un dispositif assez original jouant le rôle d'exutoire d'orage (fig. 5).

Dans sa partie aval, cet égout a une section de $3 \mathrm{~m}^{2}$, une pente de $11,4 \mathrm{~mm}$ par mètre environ, ce qui lui permet d'écouler un débit de $11 \mathrm{~m}^{3}$ environ.

Ce débit est largement supérieur au débit décennal provenant de son bassin versant (92 ha) qui, avec un coefficient de ruissellement de 0,4 , est de $4,5 \mathrm{~m}^{3}$ environ.

Mais lors d'une longue averse survenant après 
une période pluvieuse, le coefficient de ruissellement est considérablement augmenté; cet égout reçoit alors un débit beaucoup plus important qui, pour l'averse du 30 novembre 1955 , peut être évalué à $10 \mathrm{~m}^{3}$ environ (avec $\mathrm{C}=0,9$ ).

L'égout pourrait encore évacuer ce débit si le niveau de l'eau dans le Verdanson était assez bas.

Mais, dans ces Iongues averses, le Verdanson est en crue en même temps que l'égout, dont la ligne piézométrique est relevée par le niveau de l'eau dans le Verdanson. C'est ainsi que pour la crue du 30 novembre 1955 , la pente hydraulique de l'égout aurait été réduite à $8 \mathrm{~mm}$ par mètre environ, s'il n'y avait pas eu débordement de l'égout, et le débit possible de celui-ci à $9 \mathrm{~m}^{3}$ environ.

Cet égout devenait donc insuffisant pour écouler le débit de son bassin versant. Il en est résulté des engorgements, des débordements et même des jaillissements comme celui qui s'est produit à l'angle de la rue Bonnard et du faubourg Saint-Jaumes.

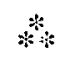

Pour améliorer cette situation, il convient d'abaisser, pour un débit donné, le niveau de crue du Verdanson, et, dans ce but, il est nécessaire :

$1^{\circ}$ De reconstruire les nombreux ponts insuffisants dont les remous successils s'ajoutent, au moins partiellement;

$2^{\circ}$ De calibrer le lit du Verdanson dans la traversée de l'agglomération et à l'aval de celle-ci jusqu'à son confluent avec le Lez.

Le problème de l'écoulement des crues sur le littoral du Languedoc méditerranéen est un problènıe difficile pour les raisons suivantes: $1^{\circ}$ Les averses dangereuses sont très longues avec des intensités élevées pendant la phase violente;

$2^{\circ}$ Les pentes insuffisantes de la zone littorale succèdent brusquement à de fortes pentes dans la zone montagneuse;

3" Pendant ces périodes pluvieuses, le niveau de la mer est le plus souvent gonflé par l'effet des vents qui soufflent du large.

C'est ainsi que le 30 novembre 1955, le nireau de la mer, par rapport au zéro des basses mers, qui était à la cote 0,47 à 18 heures, est monté à 0,62 à 24 heures.

La mer « retient», comme on dit dans le pays, ce qui ralentit l'écoulement des rivières.

Certes, d'autres pays sont également plats, mais la Hollande subit des précipitations moins violentes et peut profiter du jeu de la marée pour l'écoulement de ses excès d'eau.

Dans le Languedoc méditerranéen, pour les crues provenant des hautes vallées ou des bassins moyens, le seul remède paraît être le réservoir de crue, comme celui projeté sur l'Hérault à Saint-Guilhem-le-Désert, même si la cuvette de ce réservoir n'est pas étanche.

Pour les crues prenant naissance sur le littoral même, comme c'est le cas de l'averse du 30 novembre 1955 , il faut calibrer les rivières et les ruisseaux avec des sections compensant le défaut de pente, et en certains points ouvrir des graus de secours pour les crues.

A cet égard, les étangs du littoral jouent un rôle ulile comme champ d'expansion des' crues.

Une surélévation de $0,10 \mathrm{~m}$ du niveau des étangs de l'Arnel et de Pérols qui encadrent le Lez (superficie globale 1.275 ha) permet l'emmagasinement d'un volume de $1.275 .000 \mathrm{~m}^{3}$ correspondant au volume tolal débité par le Verdanson pendant $13 / 4 \mathrm{~h}$ à raison de $200 \mathrm{~m}^{3}$ par seconde.

\section{DISCUSSION}

Président : M. Bengienon

\footnotetext{
M. QUesner rappelle les records battus en 1940 par le département des Pyrénées-Orientales, où il est tombé à $730 \mathrm{~m}$ d'altitude : $1,85 \mathrm{~m}$ d'eau en trois jours, dont $85 \mathrm{~cm}$ en 24 heures, et $120 \mathrm{~mm}$ pendant 20 minutes sur le versant nord de la vallée. Pendant cefte mème crue, à Vernet-les-Bains, du cóté du Canigou, il est tombé $246 \mathrm{~mm}$ d'eau en quatre heures. On arait fait relever le volume d'eau qui était tombée sur le bassin versant. En évaluant le debit par le relevé de la ligne d'eau maxima et par la methode des formules de calcul de vitesse dans des sections bien calibrẻes et non grevées de remous, il n'y avait pas moyen, en l'espèce, de faire des mesures au moulinet - on trouve, pour un bassin rersant de
}

$60 \mathrm{~km}$, un eoeffeient de ruissellement égal à l'unite. Dans la vallée du lech, pour un bassin versant de $480 \mathrm{~km}^{2}$, la determination du débit par la mème méthode a donné un débit de $8,5 \mathrm{~m}^{3}$ par lim² de surtace du bassin versant.

Environ a $7 \mathrm{~km}$ a l'amont du point ou l'on a évalue le débit précédent, on est arrivé à mesurer un débit de $6.300 \mathrm{~m}^{3}$, alors qu'à l'aval il est de f'ordre de 3.700 . Le débit de $6.300 \mathrm{~m}^{3}$ était un débit de débâcle a l'aval d'un pont embaclé dont l'unique pile a éte emporté.

Il est recommandé d'être prudent dans le calcul des evacuateurs de crues pour ces pays, puisque, pratiquement, on arrive prescgue à doubler le debit maximum maximorum de la crue. 
M. Deymí observe qu'une hauteur de pluie de $240 \mathrm{~mm}$ en 4 heures correspond à une intensité de $1 \mathrm{~mm}$ par minute, ce qui est considérable pour une longue durée. Du reste, une hauteur de pluie de $240 \mathrm{~mm}$ représente le tiers de la précipitation annuelle à Montpellier. A Paris, pour la fréquence décennale, une intensité de pluie de $1 \mathrm{~mm}$ par minute correspond à une averse d'une durée de 30 minutes, tandis que, dans l'Hérault, et peut-être aussi dans les Pyrénées-Orientales, une intensité de $1 \mathrm{~mm}$ par minute correspond, pour la même fréquence décennale, à une averse d'une durée de 60 minutes. Mais il peut $y$ avoir des averses d'une durée supérieure à 1 heure avec une intensité de $1 \mathrm{~mm}$ par minute. Ces longues averses exceptionnelles ont une fréquence supérieure à 10 années.

M. Dexmé continue en ces termes :

«Ainsi que je l'ai indiqué, les averses peuvent atteindre pendant une durée de 10 ou 20 minutes des intensités plus considérables $(1,75 \mathrm{~mm}$ et jusqu'à $2 \mathrm{~mm}$ par minute). Mais ces averses, très violentes mais courtes, n'ont qu'une influence très limitée sur les crues. En effet le temps de concentration intervient et même pour une petite rivière comme le Verdanson le temps de concentration est voisin de 1 heure. Dans les violentes averses, l'eau ruisselle à une vitesse que l'on peut évaluer grossièrement à $4 \mathrm{~m}$ par seconde, soit un peu plus de $14 \mathrm{~km}$ par heure. Or, la longueur totale du bassin versant du Verdanson est de $20 \mathrm{~km}$ et n'atteint pas ce chiffre à Montpellier. La goutte la plus éloignée a donc mis un peu plus de 1 heure pour atteindre la rivière à Montpellier et c'est à partir de ce moment que s'établit le régime permanent entre le débit tombé du ciel et le débit de la rivière. Il faut donc, dans le cas du Verdanson, une averse ayant une durée d'au moins 1 heure a intensité constante pour qu'un régime permanent s'établisse. 》

Répondant à une question de M. Mostermann sur les pertes par évaporation, M. Dexmé indique qu'il ne peut pas donner de renseignements précis, mais que dans ces longues averses l'évaporation ne paraît pas importante par rapport aux quantités d'eau. tombées, d'autant plus que ces longues averses se produisent presque toutes en automne.

\section{Grande administration A.-O.F. recherche plusieurs INGENIEURS HYDRAULIQUE souterraine, agricole, navigation, confirmés et débutants.}

Ecrire HAVAS, Dakar EL 222, B. P. 503. Donner curriculum vitae, références. 